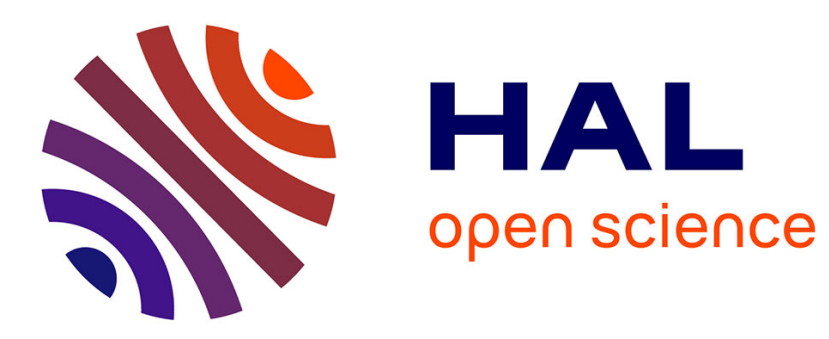

\title{
Optimal Production Facility Allocation for Failure Resilient Critical Infrastructures
}

Yi-Ping Fang, Enrico Zio

\section{To cite this version:}

Yi-Ping Fang, Enrico Zio. Optimal Production Facility Allocation for Failure Resilient Critical Infrastructures. ESREL 2013, Sep 2013, Amsterdam, Netherlands. pp.1-8. hal-00838640

\section{HAL Id: hal-00838640 \\ https://hal-centralesupelec.archives-ouvertes.fr/hal-00838640}

Submitted on 26 Jun 2013

HAL is a multi-disciplinary open access archive for the deposit and dissemination of scientific research documents, whether they are published or not. The documents may come from teaching and research institutions in France or abroad, or from public or private research centers.
L'archive ouverte pluridisciplinaire HAL, est destinée au dépôt et à la diffusion de documents scientifiques de niveau recherche, publiés ou non, émanant des établissements d'enseignement et de recherche français ou étrangers, des laboratoires publics ou privés. 


\title{
Optimal Production Facility Allocation for Failure Resilient Critical Infrastructures
}

\author{
Yi-Ping Fang, Nicola Pedroni \\ Chair on Systems Science and the Energetic challenge, Ecole Centrale Paris and Supelec, France \\ Enrico Zio \\ Chair on Systems Science and the Energetic challenge, Ecole Centrale Paris and Supelec, France \\ Energy Department, Politecnico di Milano, Italy
}

\begin{abstract}
Large scale outages on real-world critical infrastructures (CIs), although infrequent, are increasingly disastrous to society. In this paper, we model CIs as complex networks and consider the problem of facility allocation on nodes of the network for maximizing the efficiency of supply to other nodes and the resilience of the overall network in resisting to cascading failures. Of course, the investment costs for the allocation must remain limited. The framework of facility allocation optimization is originally applied to the $400 \mathrm{kV}$ French power transmission network, for allocating power generation to service the existing buses. The problem is combinatorial and multi-objective in nature, and for its solution we use the non-dominated sorting binary differential evolution (NSBDE) algorithm.
\end{abstract}

\section{INTRODUCTION}

Our modern society has come to depend on largescale critical infrastructures (CIs) to deliver resources to consumers and businesses in an efficient manner. These CIs are complex networks of interconnected functional and structural elements. Large scale outages on these real-world complex networks, although infrequent, are increasingly disastrous to society, with estimates of direct costs up to billions of dollars and inestimable indirect costs. Typical examples include blackouts in power transmission networks (USCA 2004, UCTE 2007, Pidd 2012), financial bankruptcy (Battiston et al. 2007), telecommunication outages (Newman et al. 2002), and catastrophic failures in socio-economic systems (Zhao 2011 \& Kempe 2003).

Research regarding modeling, prediction and mitigation of cascading failure in complex networks has tried to address the problem with different aspects (Battiston et al. 2007, Newman et al. 2002, Zhao 2011, Motter \& Lai 2002, Dobson et al. 2004, Baldick et al. 2008).

Albert et al. (2004) demonstrated that the vulnerability of modern infrastructure networks (e.g., power transmission network) is inherent to their organization. Thadakamalla et al. (2004) revealed that the topology of a supply network has great impact on its resilience. In the renovation and re-design of CIs, a motivating objective should then be that of rendering them failure resilient, while operationally efficient (Boorstyn \& Franck 1977). In literature, Shao et al. (2005) proposed the shrinking and searching algorithm to maximize the network reliability of a dis- tributed access network with constrained total cost. But the intense computation of network reliability prohibits its application to large size networks. Gutfraind (2010) introduced a multi-objective optimization method for constructing cascade resilient networks based on the structure of terrorist networks. Newth \& Ash (2004) used a modified Metropolis evolutionary algorithm to evolve failure resilient networks with the objective of maximizing the average network efficiency.

In practical case, the cost of knocking down existing network and reconstructing it from scratch is prohibitive, especially for CIs like the power transmission network. A more practicable alternative is to reconfigure a small part of the network topology, e.g. by reallocation the links between production facilities and consumers.

In this paper, a new efficiency index is proposed to characterize the dynamic supplying efficiency of a network where the consumer nodes receive resources or services mostly from the nearest supplying node in operation. Formulated as a large-scale, nonlinear and combinatorial multi-objective problem, the facility reallocation problem is solved by an evolutionary algorithm, i.e., non-dominated sorting binary differential evolution (NSBDE) algorithm (Li et al. 2013).

The reminder of this paper is organized as follows. We formulate the multi-objective optimization model taking cost, network supplying efficiency and failure resilience into account in Section 2. Section 3 unveils the detailed procedure of the proposed NSBDE algorithm. Section 4 illustrates the French 
$400 \mathrm{kV}$ power transmission network case study and the results analysis. Conclusions and future work are drawn on the Section 5.

\section{OPTIMIZATION MODEL}

\subsection{Network model}

We represent a complex supply network as a weighted undirected graph $G(V, E)$ comprising a set of nodes $V=\left\{v_{1}, v_{2}, \ldots, v_{N}\right\}$ together with a set of edges $E=\left\{e_{1}, e_{2}, \ldots, e_{M}\right\}$. The network nodes are generally classified into facility supplying nodes $V_{S}$ and resources consuming nodes $V_{C}\left(V_{S} \cup V_{C}=V\right)$; we use $N_{S}$ and $N_{C}$ to indicate the cardinalities of $V_{S}$ and $V_{C}$, respectively. The structure of the network is identified by an $N \times N$ interaction matrix $W$, whose element $w_{i j}$ is 0 if node $i$ and $j$ are not connected directly; otherwise it is assigned a numerical weight by the physical distance between $i$ and $j$, which we assume directly related to the transmitting cost of the link $e_{i j}$.

We define the variables to be optimized as the links of facility allocation to the different consuming nodes:

$$
X_{i j}=\left\{\begin{array}{l}
1, \text { if } i \text { is connected with } j \text { directly } \\
0, \text { otherwise }
\end{array}\right.
$$

for all $i \in V_{S}$ and $j \in V_{C}$.

A cost is associated with each rewiring. We assume that the cost is linearly proportional to the physical length of the linkage with a coefficient $\varphi$. Besides, two constraints have to be met when reallocating the facilities: (1) each consumer node is required to connect with at least one facility node or other consumer node, to make it accessible to the supplying facilities; (2) each facility node has to connect at least with one consumer node.

\subsection{Network supplying efficiency}

Notions of network supplying efficiency attempt to quantify the value of a network in operating its service. A well-known measure of network efficiency is a version of distance-based efficiency (Latora \& Marchiori 2001). For its compotation all pairs of nodes $i \in V_{S}$, and $j \in V_{C}$ are weighted by the inverse of the distance:

$$
E(G)=\frac{1}{N_{S} N_{C}} \sum_{i \in \mathrm{V}_{S}} \sum_{j \in V_{C}} \frac{1}{d(i, j)}
$$

where $d(i, j)$ is the number of edges for unweighted network or the sum of edge weights for weighted network in the shortest path from $i$ to $j$.

This definition is able to partially characterize the topological property of a complex network. To eval- uate the dynamic supplying efficiency of a network where the consumer nodes receive resources or service mostly from the nearest supplying node in operation, a new index is here proposed:

$e_{j}=\max _{i \in V_{S}}\left\{\frac{1}{d(j, i)}\right\}$

where $e_{j}$ is characterized by the most efficient supplying channel from all the facility nodes to consumer node $j$. Similarly we have the following global network supplying efficiency by averaging across all the consumer nodes:

$$
e=\frac{1}{N_{C}} \sum_{j \in V_{C}} e_{j}
$$

It is noted that $e(G) \geq E(G)$ always holds.

\subsection{Cascading failure model and network vulnerability}

There is extensive literature on cascading failure models of complex networks (e.g., Newman et al. 2002, Kempe 2003, Motter \& Lai 2002, Dobson et al. 2004, Baldick et al. 2008, Motter 2004, Vaiman et al. 2012). Two basic types are considered: percolation cascades and capacity cascades. The former originate in Physics but is often applied to Epidemiology, where it is termed "contagious" or "epidemics" (see, e.g. Newman 2003). The capacity cascades usually applies to a capacitated network such as a power transmission system and supply chain, in which edges carry flows from facility nodes to consumer nodes. Cascades occur when initial failures of a part cause flows redistribution, overloading another part and causing it to fail as well, prompting additional parts to fail in a vicious cycle. The capacity cascading failure model is the focus in this study.

Following the work in Motter \& Lai 2002, for a given supply network, suppose that at each time step one unit of the relevant quantity, which can be information, energy, etc., is exchanged between every pair of facility nodes and consumer nodes and transmitted along the shortest path connecting them. The load (or stress) at one node is then the number of shortest paths passing through it. More precisely, the load $L_{k}$ of node $k$ is quantified by the node betweenness calculated as the fraction of the facilityconsumer shortest paths passing through that node:

$$
L_{k}=\frac{1}{N_{S} N_{C}} \sum_{j \in V_{S}}, j \in V_{C}, k \in V, i \neq j \neq k \frac{n_{i j}(k)}{n_{i j}}
$$

where $n_{i j}$ is the number of shortest paths between facility nodes and consumer nodes, and $n_{i j}(k)$ is the number of facility-consumer shortest paths passing though node $k$. 
The capacity of node $k$ is assumed to be proportional to its initial node $L_{k}$ with a network tolerance parameter $\alpha$,

$$
C_{k}=(1+\alpha) L_{k}
$$

The concept of tolerance parameter $\alpha(\alpha>0)$ could be regarded as an operating margin allowing safe operation of the component under potential load increment. The occurrence of a cascading failure is initiated by removal of a node, which in general changes the distribution of shortest paths. Then the load at a particular node can change and if it increases and exceeds its capacity, the corresponding node fails. Any failure leads to a new redistribution of loads and, as a result, subsequent failures can occur.

Using this cascading failure model, the vulnerability of network $G$ can be characterized by the fraction of network efficiency loss in a cascading failure:

$$
\operatorname{Vul}(G)=\frac{E(G)-E(\bar{G})}{E(G)}
$$

where $\operatorname{Vul}(G) \in(0,1)$ and $\bar{G}$ represents the residual network structure after a cascading failure. It should be noted that the effect of the type of initial event could be significant to the cascading failure result: the loss of a cascade triggered by the failure of a critical component could be much more severe than that originated by the failure of a normal component. Therefore, we consider the worst-case scenarios in this study, i.e. one of the top five most loaded (largest betweenness) nodes, is chosen to fail in each cascading simulation, and the result is averaged on a number of simulations.

The detailed simulation of a cascading failure proceeds as follows:

Step 1. Apply formula (5) to compute the initial load of each node for a proposed network by Floyd's shortest paths algorithm (Floyd 1962), and calculate the capacity of each node based on formula (6).

Step 2. The most loaded node is chosen as failed and thus is removed from the network.

Step 3. Recur to formula (5) and Floyd's shortest paths algorithm to recalculate the load of each working node in the network.

Step 4. Test each node for failure: for each node $k$ $(k \in N)$ of the network, if $L_{k}>C_{k}$ then node $k$ is regarded as failed and thus is removed.

Step 5. If any working node fails, return back to step 3. Otherwise, terminate the cascading simulation.

\subsection{Multi-objective optimization problem formulation}

After quantifying the cost, network efficiency and cascading failure vulnerability, the facility allocation problem is formulated as a multi-objective optimization as:

$$
\begin{aligned}
& \left\{\begin{array}{l}
\min \left\{\sum_{i \in V_{S}, j \in V_{C}} \varphi X_{i j}\right\} \\
\max \{e(G)\} \\
\min \{\operatorname{Vul}(G)\}
\end{array}\right. \\
& \text { s.t. }\left\{\begin{array}{l}
\sum_{i \in V} X_{i j}>0 \forall j \in V_{C} \\
\sum_{j \in V_{C}} X_{i j}>0 \forall i \in V_{S}
\end{array}\right.
\end{aligned}
$$

The objective function (8a) is the sum of the fixed rewiring costs; (8b) and (8c) express the operating efficiency and resilience objectives, respectively. The two constraints mentioned in Section 2.1 are enforced by formula (8e) and (8f), respectively.

Observe that the least costly facility allocation is simply that when there is no link between facilities and consumers.

\section{NON-DOMINATED SORTING BINARY DIFFERENTIAL EVOLUTION ALGORITHM}

In this section, we briefly introduce the operation procedures of the NSBDE algorithm. The standard differential evolution (DE) algorithm, initially proposed as a population-based global optimization method for real-valued optimization problems, has been found to outperform alternative optimization algorithms in various fields ( $\mathrm{Li}$ et al. 2013, Price et al. 2005, Ponsich \& Coello 2011). In order to solve the combinatorial multi-objective problem of interest, the fast non-dominated sorting, ranking and elitism techniques used in non-dominated sorting genetic algorithm-II (NSGA-II) (Deb et al. 2002) is introduced into a modified binary differential evolution (MBDE) which is a binary version of DE developed to tackle single-objective binary-coded optimization problems (Wang et al. 2010). The NSBDE proceeds as follows (Li et al. 2013):

\section{Step 1. Initialization of parameters}

Set the values of the population size $N P$, the crossover rate $C R$, the scaling factor $F$, and the maximum generations $N_{\max }$. 
Step 2. Generation of initial population and evaluation

Initialize each individual in the population which is represented as a bit-string and denoted as $p x_{i}^{t}=$ $\left\{p x_{i j}^{t}, \mid p x_{i j}^{t} \in\{0,1\} ; i=1,2, \ldots, N P, j=1,2, \ldots, M\right\}$, where $N P$ is the population size and $M$ is the dimensionality of the solutions. Each individual is also called a chromosome and forms a candidate solution to the problem. Each bit of each initial chromosome takes a value from the set $\{0,1\}$ with probability equals to 0.5 : the bit takes ' 1 ' if the corresponding facility node and consumer node are connected, ' 0 ' otherwise.

Each of the NP chromosomes is evaluated by computing the three objective functions, i.e. formula (8a), (8b) and (8c) on its corresponding network topology.

\section{Step 3. Generation of trial population}

Apply the binary tournament selection operator (Deb et al. 2002) to the population $P X^{t}$ to generate a trial population $P V^{t}$, which undergoes the evolution operations of mutation and crossover.

\section{Step 3.1 Mutation}

The following probability estimation operator $P(p x)$ is utilized to generate the mutated individuals according to the information of the parent population:

$$
P\left(p x_{i j}^{t}\right)=\frac{1}{1+e^{-\frac{2 b\left[p x_{r 1, j}^{t}+F\left(p x_{r 2, j}^{t}-p x_{r 3, j}^{t}\right)-0.5\right]}{1+2 F}}}
$$

where $b$ is a positive real constant, usually set as $6 ; F$ is the scaling factor; $p x_{r 1, j}^{t}, p x_{r 2, j}^{t}$ and $p x_{r 3, j}^{t}$ are the $j$-th bits of three randomly chosen individuals at generation $t$. According to the probability estimation vector $P\left(p x_{i}^{t}\right)=\left[p x_{i, 1}^{t}, p x_{i, 2}^{t}, \ldots\right.$ $\left.p x_{i, N}^{t}\right]$ created by Eq. (9), the corresponding offspring $p u_{i}^{t}$ of the current target individual $p x_{i}^{t}$ is generated as Eq. (10).

$$
p u_{i j}^{t}=\left\{\begin{array}{l}
1, \text { if } \operatorname{rand} \leq P\left(p x_{i j}^{t}\right) \\
0, \text { otherwise }
\end{array}\right.
$$

where rand is a uniformly distributed random number within the interval $[0,1]$.

\section{Step 3.2 Crossover}

The crossover operator is used to mix the target individual and its mutated individual. The trial individual $p v_{i j}^{t}=\left(\begin{array}{lll}p v_{i, 1}^{t}, p v_{i, 2}^{t}, \ldots & p v_{i, N}^{t}\end{array}\right)$ can be obtained by the crossover operator as follows, $p v_{i j}^{t}=\left\{\begin{array}{c}p u_{i j}^{t}, \text { if } r a n d j \leq C R \text { or } j=\text { randi } \\ p x_{i j}^{t}, \text { otherwise }\end{array}\right.$

where randj $\in(0,1]$ is a uniform random value, $C R$ is the crossover rate, and randi is a uniform discrete random number in the set $\{1,2, \ldots, N P\}$.

\section{Step 4. Evaluation}

Evaluate each of the NP chromosomes in the population $P V^{t}$ by computing its rewiring cost (8a), network supplying efficiency (8c) and its resilience to cascading failures $(8 \mathrm{~b})$ by performing the cascade process simulation procedure presented in Section 2.3.

\section{Step 5. Union and Sorting}

Combine the parent and trial populations to obtain a union population $P U^{t}=P X^{t} \cup P V^{t}$. Rank the individuals in the union population by the fast nondominated sorting algorithm (Deb et al. 2002) with respect to the objective values, and identify the ranked non-dominated fronts $F_{1}, F_{2}, \ldots, F_{k}$ where $F_{1}$ is the best front, $F_{2}$ is the second best front and $F_{k}$ the least good front.

\section{Step 6. Selection}

Select the first $N P$ individuals from $P U^{t}$ to create a new parent population $P X^{t+1}$. The crowding distance is used in this step to choose the individuals with the same front, where crowing refers to the density of solution present in a neighborhood of an individual of specified radius (Deb et al. 2002). We prefer the individual which is located in a region with least number of individuals. The algorithm stops when it reaches the predefined maximum generations $N_{\max }$.

\section{CASE STUDY AND RESULTS ANALYSES}

In this paper, the $400 \mathrm{kV}$ French power transmission network (Figure 1) is taken for exemplification of the proposed approach. The network is built from the data on the $400 \mathrm{kV}$ transmission lines of the RTE website (RTE 2011). It has 171 nodes (substations) and 220 edges (transmission lines). We distinguish the generators, which are the source of power, from the other distribution substations, that receive power and transmit it to other substations or distribute it in local distribution grids. By obtaining the power plants list from the EDF website (EDF 2013) and relating them with the ID of the buses in the transmission network, we have 26 generators and 145 distributors. Only the nuclear power plants, hydraulic 
plants and thermal power plants whose installed capacities are larger than $1000 \mathrm{MW}$, are considered.

For reallocation of the power generating nodes (facilities) to the other nodes (consumers), the NSBDE algorithm is applied. The parameters used to run the NSBDE algorithm are reported in Table 1. The network tolerance parameter $\alpha$ is set as 0.3; linkage cost parameter $\varphi$ is set as 1 in the experiment.

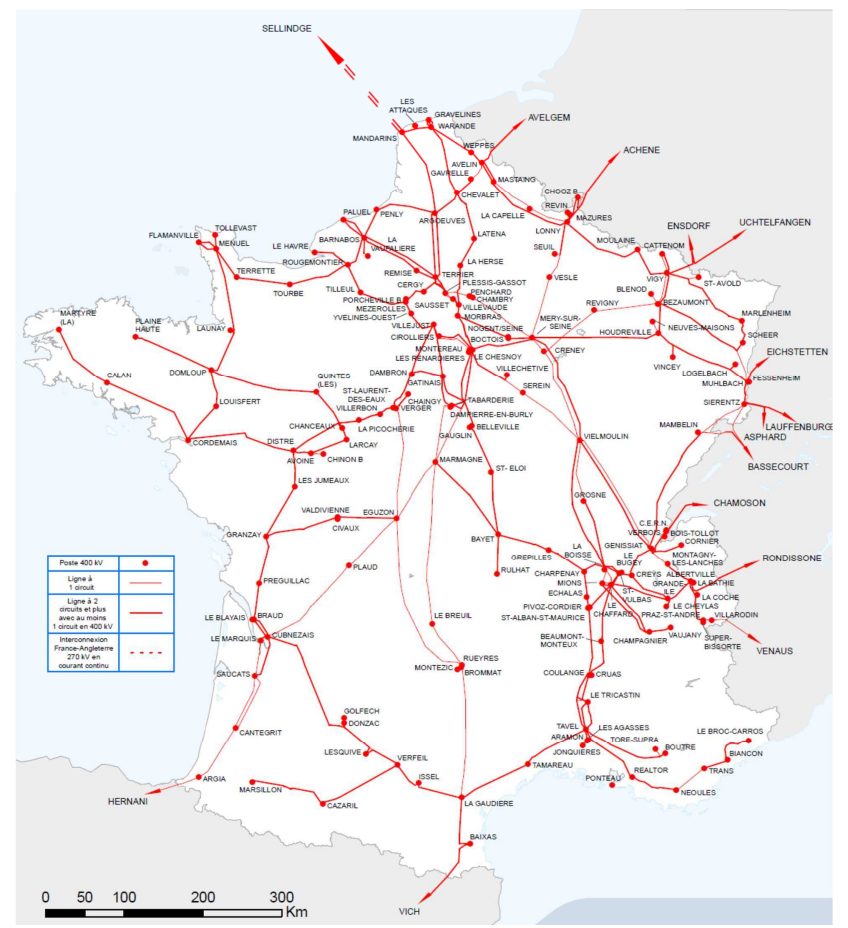

Figure 1 . The 400kV French power transmission network

Table 1. The parameters of the NSBDE algorithm.

\begin{tabular}{ll}
\hline Parameters & Values \\
\hline Population size $N P$ & 25 \\
Dimensionality of solution $M$ & 3770 \\
Crossover rate $C R$ & 0.9 \\
Scaling factor $F$ & 0.2 \\
Maximum generation $N_{\max }$ & 500 \\
\hline
\end{tabular}
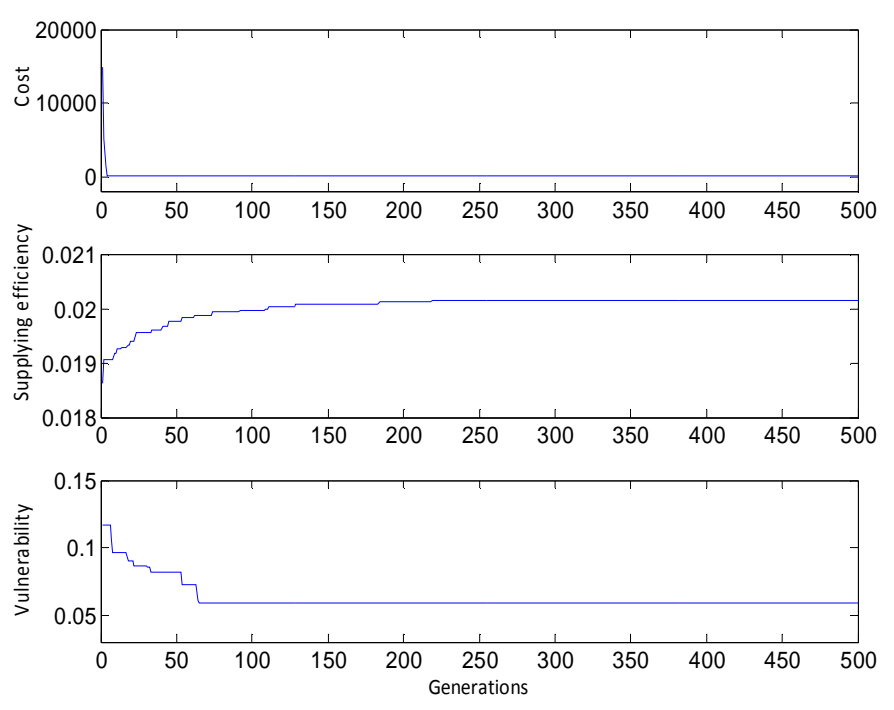

Figure 2. The convergence plots of objective finesses during the evolution of NSBDE
Figure 2 reports the convergence plots of one experiment run of the NSBDE algorithm. The three panels show the three optimal solutions with regard to the three objectives, respectively. It is observed that the algorithm is able to converge after about 250 generations.

The Pareto dominance front obtained by the NSBDE algorithm at convergence is illustrated in the 3-D space of Figure 3(a). The three 2-D projections of the solutions are shown in Figure 3(b), 3(c) and 3(d), respectively. The square point in Figure 4 represents the true network with the links at present, which is also the least costly network; the star point is the most resilient network, whose cascading vulnerability is 0.074 , and the diamond point represents the network of most supplying efficient, 0.204. It is not unexpected that the original network is the least costly one, since the electrical transmission lines and substations are placed with geographical constraint and connections between two distant substations are avoided in.

Figure 3(c) shows that the cascading resilience of the $400 \mathrm{kV}$ French power transmission network is

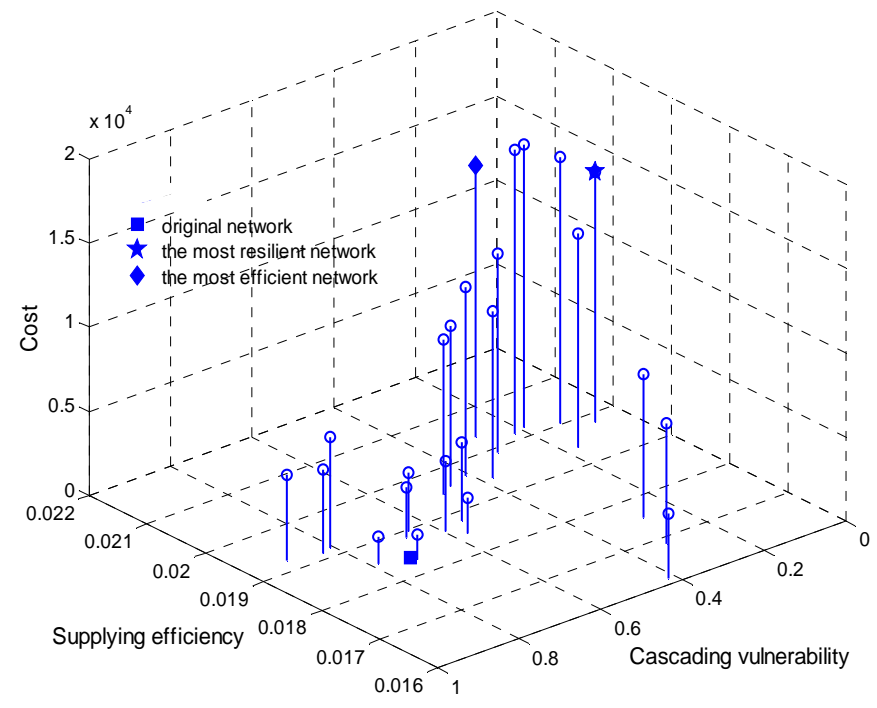

(a)

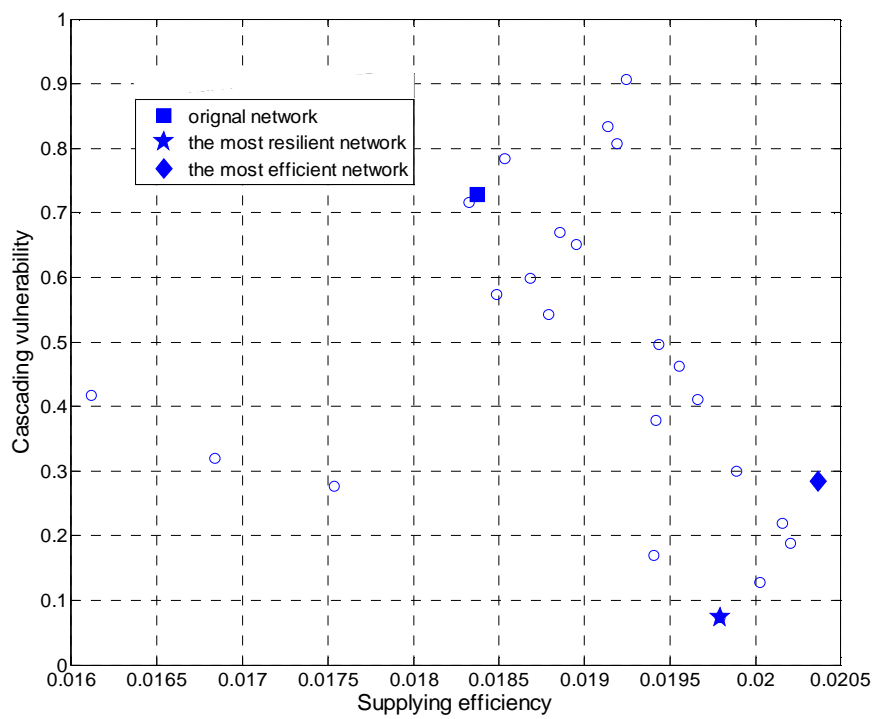

(b) 


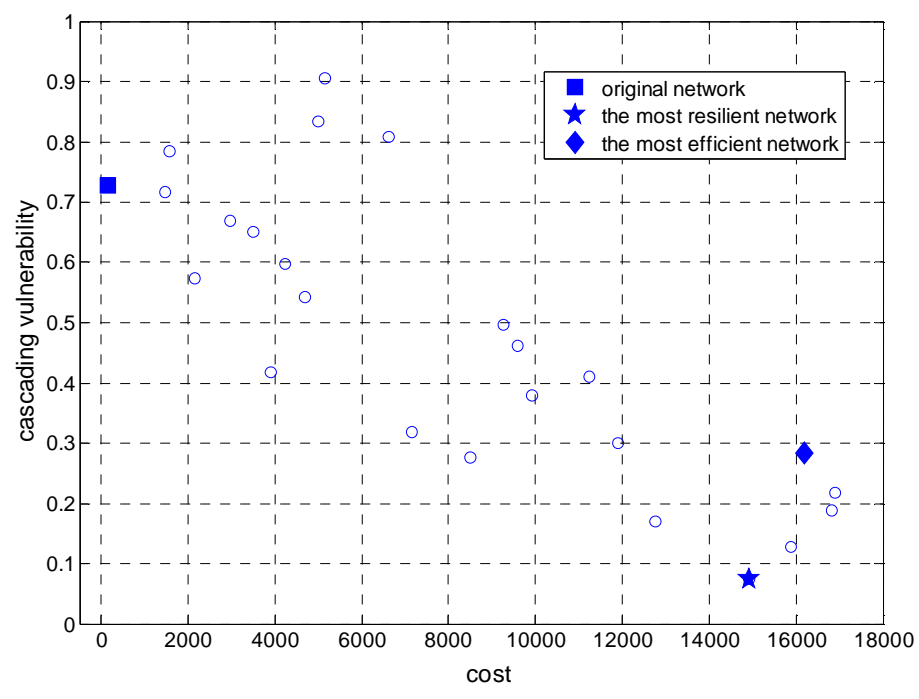

(c)

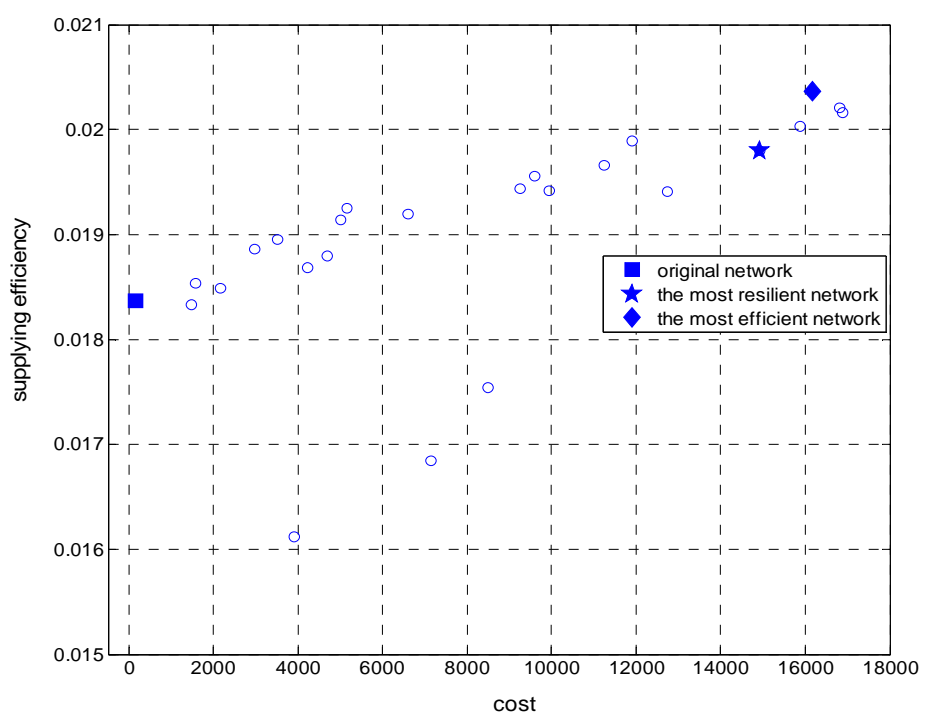

(d)

Figure 3. Pareto front in 3-D space and its 2-D projections reached by a population of 25 chromosomes evolving for 500 generations

improved significantly by properly rewiring the generator-distributor connections, though at a cost; the network vulnerability is decreased from 0.728 to 0.074 (when $\alpha=1.3$ ) with an increased average cost of $1.5 \times 10^{4}$. Figure 4 reports the cascading vulnerability comparison between the original network and the most resilient one with different tolerance parameters. It shows that when the network tolerance is very low, i.e. $0<\alpha<0.1$, the optimized network loses most of its efficiency, i.e., it is quite vulnerable to targeted attacks. However, when $\alpha \geq 0.3$ (which is generally the normal operating condition Baldick 2008), the optimized network loses less than $10 \%$ of its efficiency during a cascading failure initiated by intentional attack.

The inner figure of Figure 4 shows that the connection of the most resilient network is much denser than the original network. Given that it is not easy to realize a physical connection between two distant substations, a careful trade-off between cost and cascading resilient improvement should be taken into account by the decision makers.

It is noted from Figure 3(d) that the supplying efficiency of the network can also be increased, although in a limited range. According to its definition in Eq. (4), one cannot improve the supplying efficiency any more as long as a distributor has connected with its nearest generator, which is normally the case for most of the distributors in the real network. Therefore, we remove the supplying efficiency objective, i.e. keep only the network resilience and cost objectives for the optimization. .

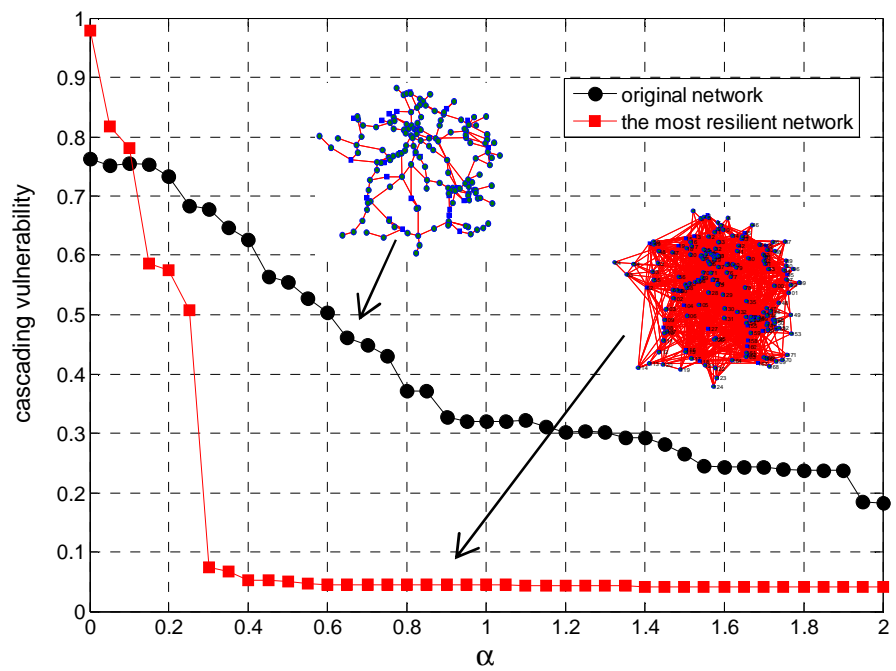

Figure 4. Comparison of the cascading vulnerability between the original and the most resilient networks under different network tolerance

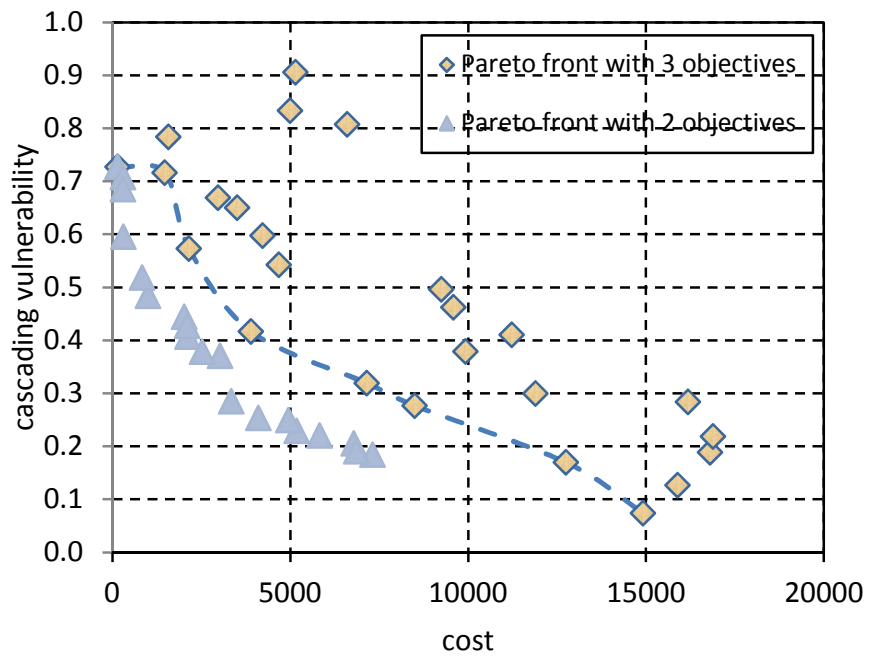

Figure 5. Comparison of the Pareto dominance fronts between three and two objectives optimization

Figure 5 compares the Pareto fronts obtained by two-objectives (reallocation cost and cascading resilience) and three-objective (reallocation cost, supplying efficiency and cascading resilience) NDBDE optimization in the cascading vulnerability-cost space. The parameter settings of the two-objective optimi- 
zation are of the same as for the three-objective optimization. One can find that the solutions from twoobjective optimization are obviously better than those of three-objective optimization.

Figure 6 reports the comparison of the topology of the original network and a network corresponding to a two-objective optimization solution $(310.6,0.59)$ when $\alpha=1.3$. The links difference between the two networks is 10 , i.e. only 10 links are required to be rewired for the original network to gain a $19.2 \%$ cascading resilience improvement (the cascading vulnerability is decreased from 0.73 to 0.59 ). Besides, the optimized network gains a slight supplying efficiency improvement, i.e. from 0.0184 to 0.0186 .

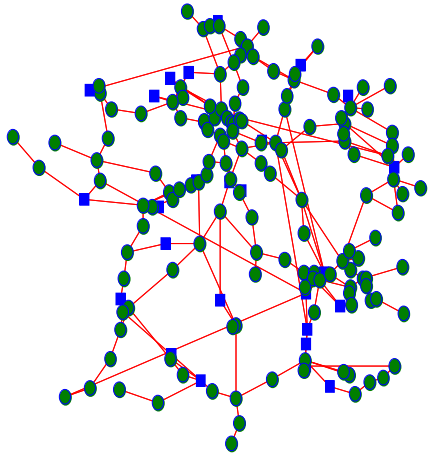

(a)

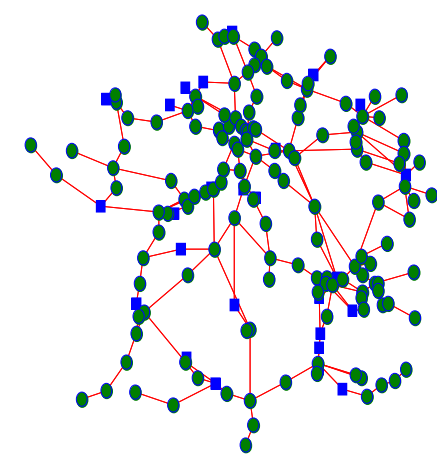

(b)
Figure 6. Comparison of the topology of the original network and a network corresponding to one of the 2-objectives optimization solutions $(310.6,0.59)$; square nodes are generators and circle nodes are distributors.

As mentioned before, a tradeoff between the cost and resilience improvement should be taken into account in decision-making. Along the Pareto frontier of the potential solutions, there are some points at which a small sacrifice of cost gives a large gain of cascading resilience. More generally, by taking a network solution and its neighbor on the frontier (the less costly one), one can define a rate of change of cascading resilience with respect to cost: $|\Delta V u l|$ $\Delta$ cost $\mid$. This rate can be utilized as a reference to choose the optimized network: the larger the ratio, the more preferred the network is.

\section{CONCLUSIONS AND FUTURE WORK}

In many CIs, the structure emerges through an unplanned growth process to meet service demand and/or results from optimization of costs. However, the increasing threat of large scale failures makes it vital to think of the design of resilient network systems capable to resist against and recover from cascading failures.

In this paper, the problem of allocating of production facilities to consumers nodes by rewiring links has been considered under the objectives of maxim- izing the network cascading failure resilience and supplying efficiency, while maintaining the investment costs limited. Exemplification has been done by taking the $400 \mathrm{kV}$ French power transmission network as an illustration. In realistic cases of networks connecting a large number of nodes, the problem is a combinatorial multi-objective optimization problem. We effectively tackled this by the proposed NSBDE multi-objective algorithm, within a Pareto optimality scheme of search for non-dominated solutions. The results of the case study show that facility allocation can be optimized to improve the cascading resilience of a supplying network system at an acceptable cost.

The analysis performed focuses only on the topological and geographical distance features of the network, thus neglecting important physical characteristics like: (i) the "electrical" length of a path differs from the topological, depending on the difficulty (impedance for high voltage transmission lines) of transmission; (ii) the electrical power flow is not necessarily routed through the shortest path; rather the transmission of power is completely determined by physical rules, e.g., Kirchoff's laws, nodal voltages etc. (iii) the capacity of electrical generation and the amount of local consumption are heterogonous for different components in the power transmission network. Establishing effective ways of bringing these physical characteristics into the topological analysis forms the possible future work in this thread of investigation.

\section{REFERENCES}

Albert, Réka, István Albert, and Gary L. Nakarado 2004. Structural vulnerability of the North American power grid. Physical Review E 69(2): 025103.

Baldick, R., et al. 2008. Initial review of methods for cascading failure analysis in electric power transmission systems IEEE PES CAMS task force on understanding, prediction, mitigation and restoration of cascading failures. Power and Energy Society General Meeting-Conversion and Delivery of Electrical Energy in the 21 st Century IEEE: 1-8.

Battiston, S., Delli Gatti, D., Gallegati, M., Greenwald, B., \& Stiglitz, J. E. 2007. Credit chains and bankruptcy propagation in production networks. Journal of Economic Dynamics and Control 31(6), 2061-2084.

Boorstyn, R., \& Frank, H. 1977. Large-scale network topological optimization. Communications, IEEE Transactions on 25(1): 29-47.

Deb, K., Pratap, A., Agarwal, S., \& Meyarivan, T. A. M. T. 2002. A fast and elitist multiobjective genetic algorithm: NSGA-II. Evolutionary Computation, IEEE Transactions on 6(2): 182-197.

Dobson, I., Carreras, B. A., Lynch, V. E., \& Newman, D. E. 2004. Complex systems analysis of series of blackouts: cascading failure, criticality, and self-organization. Bulk power system dynamics and control-VI: 22-27. 
EDF 2013. En direct de nos centrales. Retrieved Avril 2013, http://france.edf.com/france-45634.html.

Floyd, R. W. 1962. Algorithm 97: shortest path. Communications of the ACM 5(6): 345.

Gutfraind, A. 2010. Optimizing topological cascade resilience based on the structure of terrorist networks. PloS one 5(11): e13448.

Kempe, D., Kleinberg, J., \& Tardos, É. 2003. Maximizing the spread of influence through a social network. In Proceedings of the ninth ACM SIGKDD international conference on Knowledge discovery and data mining ACM: 137-146.

Latora, V., \& Marchiori, M. 2001. Efficient behavior of smallworld networks. Physical review letters 87(19): 198701.

Li, Y. F., Sansavini, G., \& Zio, E. 2013. Non-Dominated Sorting Binary Differential Evolution for the Multi-Objective Optimization of Cascading Failures Protection in Complex Networks. Reliability Engineering \& System Safety 111: 195-205.

Motter, A. E., \& Lai, Y. C. 2002. Cascade-based attacks on complex networks. Physical Review E 66(6): 065102.

Motter, A. E. 2004. Cascade control and defense in complex networks. Physical Review Letters 93(9): 098701.

Newman, M. E., Forrest, S., \& Balthrop, J. 2002. Email networks and the spread of computer viruses. Physical Review E 66(3), 035101.

Newman, M. E. 2003. The structure and function of complex networks. SIAM review 45(2): 167-256.

Newth, D., \& Ash, J. 2004. Evolving cascading failure resilience in complex networks. In Proc. of 8th Asia Pacific Symp. on Intelligent and Evolutionary Systems. December 2004.

Pidd H. 2012. India blackouts leave 700 million without power. The Guardian 31 July 2012.

Price, K. V., Storn, R. M., \& Lampinen, J. A. 2005. Differential evolution a practical approach to global optimization.

Ponsich, A., \& Coello, C. A. 2011. Differential Evolution performances for the solution of mixed-integer constrained process engineering problems. Applied Soft Computing 11(1): 399-409.

RTE 2011. Le Réseau de Transport d'Electricité $400 \mathrm{kV}$. http://www.rte-france.com.

Shao, F. M., Shen, X., \& Ho, P. H. 2005. Reliability optimization of distributed access networks with constrained total cost. Reliability, IEEE Transactions on 54(3): 421-430.

Thadakamaila, H. P., Raghavan, U. N., Kumara, S., \& Albert, R. 2004. Survivability of multiagent-based supply networks: a topological perspect. Intelligent Systems, IEEE 19(5): 24-31.

USCA 2004. Final Report on the August 14, 2003 Blackout in the United States and Canada, US-Canada Power System Outrage Task Force, Tech. Rep.

UCTE 2007. Final Report System Disturbance on 4 Nov. 2006, Union for the Coordination of Transmission of Electricity, Tech. Rep.

Vaiman, M., Bell, K., Chen, Y., Chowdhury, B., Dobson, I., Hines, P., ... \& Zhang, P. 2012. Risk assessment of cascading outages: Methodologies and challenges. Power Systems, IEEE Transactions on 27(2): 631-641.

Wang, L., Fu, X., Menhas, M. I., \& Fei, M. 2010. A modified binary differential evolution algorithm. In Life System Modeling and Intelligent Computing: 49-57. Springer Berlin Heidelberg.

Zhao, K., Kumar, A., Harrison, T. P., \& Yen, J. 2011. Analyzing the resilience of complex supply network topologies against random and targeted disruptions. Systems Journal, IEEE 5(1), 28-39. 